\title{
EDITORIAL
}

\section{Celebrating the 2015 Nobel Prize in Physiology or Medicine of Dr Satoshi Ōmura}

The Journal of Antibiotics (2016) 69, 1; doi:10.1038/ja.2015.113; published online 21 October 2015

\begin{abstract}
A s Editor-in-Chief, I would like to congratulate Dr Satoshi Ōmura, A Distinguished Emeritus Professor at Kitasato University in Japan, and Emeritus Editor-in-Chief of this journal, who has been awarded the 2015 Nobel Prize in Physiology or Medicine for 'discoveries concerning a novel therapy against infections caused by roundworm parasites'. He shares the prize with his primary collaborator on the discovery and development of the drug ivermectin, Prof. William Campbell, as well as with Youyou Tu for her work on artemisinin, the widely used antimalarial.

Dr Ōmura's discoveries in natural products chemistry and medicinal chemistry are legion. They have been extensively cited around the world, and used in the titles of about 1200 research papers every year. His profound contributions in the field impressively span the areas of microbiology, isolation and structural elucidation, biosynthesis, biological investigation and chemical synthesis. These contributions are described in over 1000 publications and many books and patents.

Over a 50-year career, Dr Ōmura has discovered over 180 distinct types and more than 480 novel bioactive compounds, many playing significant roles in medicine, industry or as important reagents, including staurosporine and lactacystin. However, the most unique and most significant compound has been avermectin.
\end{abstract}

Avermectin and its dihydro derivative, ivermectin, are certainly among his best-known discoveries. Ivermectin, in particular, has been used extensively for human and animal health as the world's leading anthelmintic agent, as well as for agricultural purposes. Millions of people have been saved from blindness and disfiguration by this drug, and millions more are expected to benefit from it as its uses expand into new domains such as strongyloidiasis and scabies. The WHO is currently overseeing global disease elimination programmes for onchocerciasis and lymphatic filariasis primarily using ivermectin, both of which are close to accomplishing their goal.

The full list of natural products discovered in Ōmura's laboratories is also very impressive. This inspirational aspect of his work cannot be underestimated, for it fuels the field of chemical synthesis with opportunities to discover and invent further concepts in chemistry, biology and medicine.

The impact of Ōmura's work extends beyond the field of antibiotics and into the world of science.
Kuniaki Tatsuta

Waseda University, Tokyo, Japan E-mail: tatsuta@waseda.jp 